This item was submitted to Loughborough's Research Repository by the author.

Items in Figshare are protected by copyright, with all rights reserved, unless otherwise indicated.

\title{
Testing of an anti-soiling coating for PV module cover glass
}

PLEASE CITE THE PUBLISHED VERSION

https://doi.org/10.1109/PVSC.2018.8547272

PUBLISHER

(c) IEEE

VERSION

AM (Accepted Manuscript)

\section{PUBLISHER STATEMENT}

This work is made available according to the conditions of the Creative Commons Attribution-NonCommercialNoDerivatives 4.0 International (CC BY-NC-ND 4.0) licence. Full details of this licence are available at: https://creativecommons.org/licenses/by-nc-nd/4.0/

\section{LICENCE}

CC BY-NC-ND 4.0

\section{REPOSITORY RECORD}

Isbilir, Kenan, Fabiana Lisco, Gerald Womack, Ali Abbas, and Michael Walls. 2019. "Testing of an Anti-soiling Coating for PV Module Cover Glass". figshare. https://hdl.handle.net/2134/34336. 


\title{
Testing of an Anti-Soiling Coating for PV Module Cover Glass
}

\author{
Kenan Isbilir, Fabiana Lisco, Gerald Womack, Ali Abbas and John Michael Walls \\ CREST, Wolfson School of Mechanical, Electrical and Manufacturing Engineering, Loughborough \\ University, Loughborough, Leicestershire, LE11 3TU, UK
}

\begin{abstract}
Soiling of solar module cover glass can significantly reduce the module power output. Coatings can be applied to the cover glass surface to reduce adhesion and make the surfaces easier to clean. These coatings should be resilient and resistant to environmental damage. A hydrophobic anti-soiling coating was exposed to a variety of environmental and abrasion stress tests. The hydrophobic performance of the coating was measured by monitoring the water contact angle and the water roll off angle after exposure to a range of environmental and mechanical stress tests. The coating was shown to be highly resistant to damp heat and thermal cycling. However, it was degraded by UV exposure and damaged during abrasion tests. The coating was also exposed to outdoor testing to compare the laboratory results with real performance degradation.
\end{abstract}

Index Terms - anti-soiling coating, hydrophobic coating, water contact angle, coating degradation

\section{INTRODUCTION}

Soiling attenuates light into the solar absorber reducing the power output of PV arrays, with a decrease in the range 15$30 \%$ reported for moderate dust accumulation [1]. Various techniques can be used to reduce soiling. Using water for cleaning will work but in areas such as deserts, sourcing water is difficult and expensive. Robotic cleaning methods may damage the glass and in most cases will require water based cleaning solutions. Anti-soiling coatings are being used as a way of reducing soiling or at least making them easier to clean. These coatings can be either hydrophilic or hydrophobic. Hydrophilic coatings have a high surface energy and provide a cleaning action by spreading the water. These 'self-cleaning' coatings are claimed to provide 'active' cleaning and are assisted by a photoactive $\mathrm{TiO}_{2}$ layer, which breaks down organic chains, making the surface easier to wash. However, due to the presence of $\mathrm{TiO}_{2}$, the reflectivity of the cover glass is increased [2].

The terminology 'hydrophobic' means water repellent and it generally refers to surfaces with a high water contact angle $\left(>90^{\circ}\right)$. The contact angle of a water droplet can be calculated using Young's equation [3]. The dependence of surface wettability on the roughness of the surface is described by the Wenzel state [4]. A Cassie-Baxter state [5] occurs when vapour is present at the water-surface interface due to surface roughness. Hydrophobic surfaces have low surface energy. This provides less adhesion for soiling on the surface. It also causes water droplets to roll off the surface more easily and carry away dust and other forms of soiling.
Hydrophobic coatings are already used in a variety of applications and are beginning to be used on solar modules. Their use is ubiquitous in the ophthalmic industry where hydrophobic coatings are applied to spectacle lenses to make them easier to clean. This application has similar requirements to solar cover glass such as high optical transmission, high water contact angle and excellent durability. The ophthalmic industry has developed test methods to evaluate the durability of hydrophobic coatings for use on spectacle lenses. Stress tests used for PV module qualification standards are also commonly used to simulate environmental durability. In this work, we have tested the use of a commercially available hydrophobic coating that is widely used on lenses and displays to make the surfaces easy to clean. We have used a range of laboratory test methods that we have identified previously [6]. These tests have been used to identify any durability issues and associated degradation mechanisms. In addition, coated glass surfaces were exposed to outdoor testing for six months. This study has enabled us to identify a laboratory test that induces a performance loss similar to that observed outdoors.

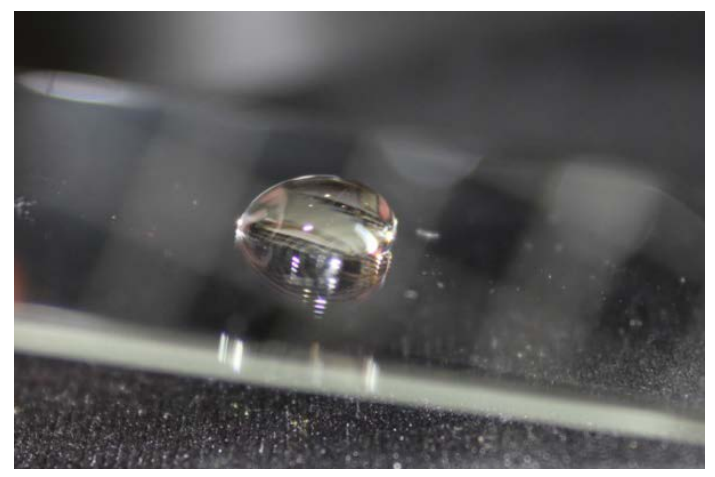

Fig. 1. Water drop on hydrophobic coated glass surface.

\section{EXPERIMENTAL}

\section{A. Performance Parameters}

Hydrophobic coatings have low surface energy which reduces adhesion to soiling. Although this does not prevent soiling, it reduces the extent and makes the surfaces easier to clean. The parameters that determine the performance of a hydrophobic coating are water contact angle (WCA) and Rolloff Angle (RoA). Materials which have a water contact angle greater than $90^{\circ}$ are considered to be hydrophobic. However, for anti-soiling applications, it is necessary that the coatings 
also have a low roll-off angle. This will ensure that water droplets roll off the module surface even at a slight installation angle. The best performing coatings are those with the highest water contact angle and the lowest water roll-off angle.

It may be necessary to compromise between the hydrophobic performance and the other properties of the coating [1]. Zero optical losses are a requirement for use on the cover glass for PV applications. The hydrophobic coating used in this study is $20 \mathrm{~nm}+/-5 \mathrm{~nm}$ thick. However, it may be possible to tune the optical properties of a thicker coating to also produce anti-reflective effect [7]. This would increase the energy generation of the module and improve the overall financial return.

\section{B. Methodology}

The hydrophobic coatings were deposited on $1 \mathrm{~mm}$ thick glass surfaces using two techniques. One set was deposited by thermal evaporation under vacuum. The second set was sprayed from solution in air. The performance of the two sets of coatings was comparable. In this work, the results of samples deposited by evaporation are presented. UV exposure, damp heat, humidity freeze, thermal cycling, solubility and abrasion resistance tests were used to assess the coating durability.

All surfaces were visually checked before exposure to each test. Intermediate checks were performed to monitor the degradation pattern during exposure. A Varian Cary ${ }^{\circledR}$ UV 5000 spectrophotometer was used for transmittance and reflectance measurements. A Dataphysics OCA 20 contact angle measurement system was used for water contact angle measurements. A bespoke device was developed to measure the water roll-off angle. A Thermo Scientific K-Alpha XPS surface analysis tool was used to study the changes in chemical composition of the surface and to determine the cause of degradation during testing. A Scanning Electron Microscope (SEM - Jeol ${ }^{\circledR} 7100 \mathrm{~F}$ and Leo 1530 VP FEG-SEM) and a Transmission Electron Microscope (TEM - Tecnai F20) were used to image the surface and cross section of the coating before and after testing, demonstrating the effect of each stress test on the coating itself and on the nanoparticle size distribution.

\section{RESULTS}

\section{A. Nanoparticle size Distribution}

The SEM analysis showed the presence of nanoparticles (NPs) dispersed on the surface of the as received samples, as shown in Figure 2a. The NPs size distribution was estimated by using the Image J software. The NPs distribution is found to be $1.06 \mathrm{e}+8 \mathrm{NPs} / \mathrm{cm}^{2}$ with the mean particle size (xc) $\sim 36 \mathrm{~nm}$. Figure 3 shows the occurrence frequency and diameter of the NPs. The STEM analysis confirms the presence of the nanoparticles placed on the surface of the coating in Figure $2 \mathrm{~b}$.

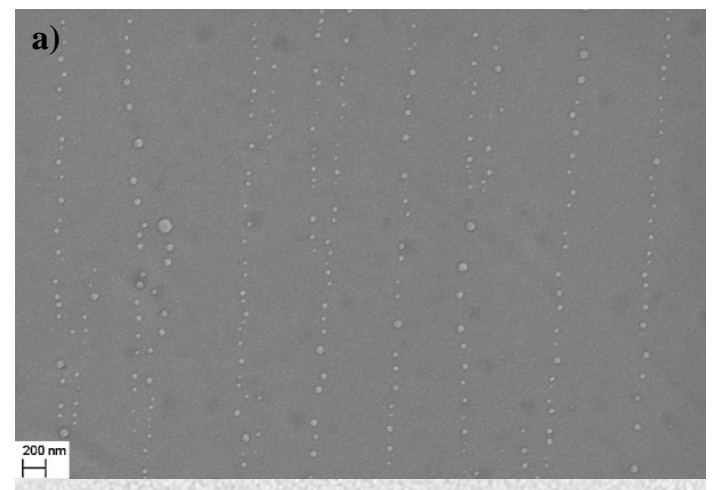

b)

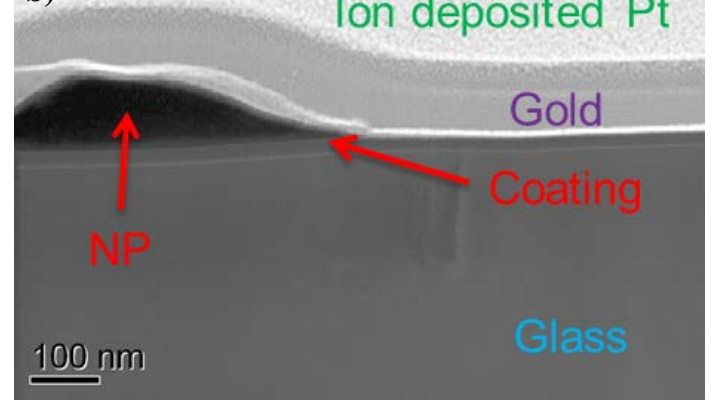

Fig. 2. a) NPs are visibly dispersed on hydrophobic coating in the SEM planar view image b) STEM cross-sectional image of the coating.

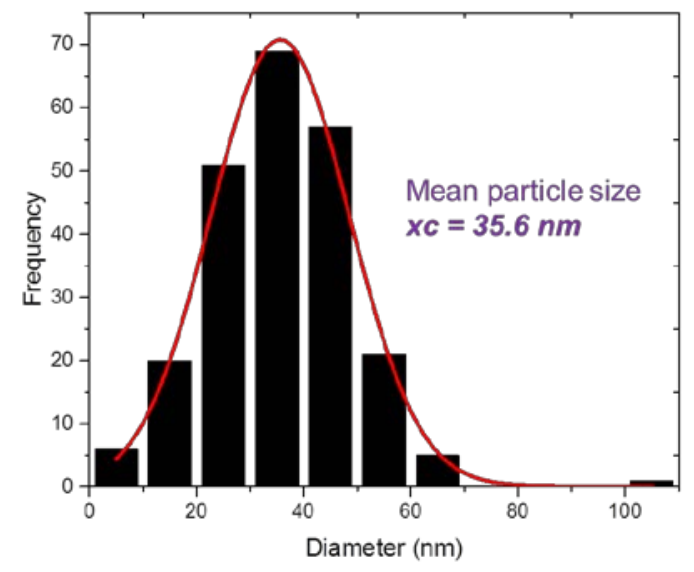

Fig. 3. Representative NPs size distribution estimated on the SEM captured sample area in Figure 2a.

\section{B. Long-term Exposure to High Temperature and Humidity}

Surfaces were exposed to the damp heat test at $85^{\circ} \mathrm{C} / 85 \%$ relative humidity (RH) for 4000 hours. Intermediate checks were performed after 250, 500 and 1000 hours of exposure. Figure 4 shows the results of exposure to damp heat at 1000 hour intervals. The results showed that the coating is resilient to high temperature and humid conditions. After 4000 hours of exposure, the WCA was reduced by only $2 \%$. The water rolloff angle improved slightly, this is possibly due to the coating 
being annealed during exposure to damp heat. No effect on the optical transmittance was observed.

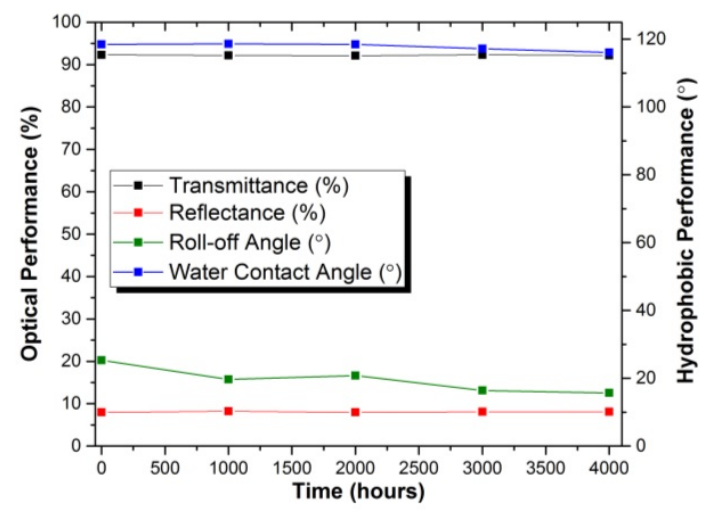

Fig. 4. Optical and hydrophobic performance during Damp Heat Exposure.

\section{Humidity Freeze Test}

Hydrophobic coated samples were exposed to 10 humidity freeze cycles. Each cycle consists of 20 hours of $85^{\circ} \mathrm{C}$ and $85 \%$ relative humidity $(\mathrm{RH})$ exposure followed by exposure to a minimum of 30 minutes at $-40^{\circ} \mathrm{C}$ with no $\mathrm{RH}$ control according to IEC 61215-2:2016 [8].

TABLE I - Humidity FREEZE TEST REsults

\begin{tabular}{|c|c|c|c|c|}
\hline & Initial & $\mathbf{1}$ cycle & $\mathbf{5}$ cycles & $\mathbf{1 0}$ cycles \\
\hline WCA ( $\left.{ }^{\circ}\right)$ & 118.1 & 118.0 & 117.4 & 117.7 \\
\hline Roll-off angle ( ${ }^{\circ}$ ) & 26.9 & 24.9 & 22.8 & 23.2 \\
\hline R (\%) & 8.2 & 8.2 & 8.2 & 8.2 \\
\hline T (\%) & 91.8 & 91.8 & 91.8 & 92.0 \\
\hline
\end{tabular}

The coatings were resilient to the humidity freeze test and showed no degradation after the exposure. The hydrophobic and optical performances were measured after 1, 5 and 10 cycles. Table I shows the results during the exposure. Transmittance and reflectance measurements were collected at $550 \mathrm{~nm}$ wavelength.

\section{Thermal Cycling Test}

A thermal cycling test procedure in accordance with IEC 61215-2:2016 [8] was used to evaluate the durability of the hydrophobic coating against stresses caused by temperature changes. Test samples were exposed to a total of 1000 cycles. Figure 5 shows the results of the thermal cycling exposure, with intermediate checks at 100 cycle intervals.

The results showed that the hydrophobic and optical performance of the coating was unaffected after 1000 cycles of thermal cycling exposure.

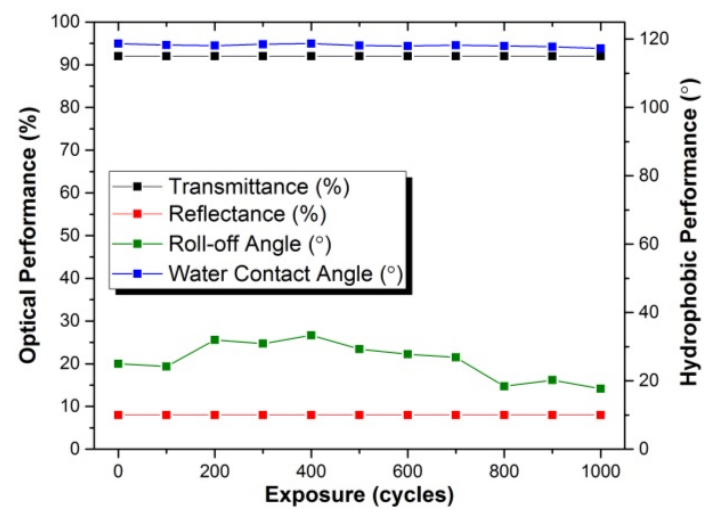

Fig. 5. Optical and hydrophobic performance before and after Thermal Cycling Exposure.

\section{E. Abrasion Resistance}

A reciprocating abraser was used to evaluate the resistance against abrasion. Felt pad and CS10 [9] were used as abrasive materials. The applied loads were $5 \mathrm{~N}$ and $10 \mathrm{~N}$ for each material. Table II shows the results before and after 50, 100, 200 and 300 cycles of abrasion using CS10 with a $10 \mathrm{~N}$ load. Figure 6 shows the differences between the WCA and RoA measurements, after CS10 and felt pad abrasion with a $10 \mathrm{~N}$ load.

After 300 cycles of abrasion with CS10, the WCA was significantly reduced. The RoA was increased to $47.1^{0}$ after 200 cycles but a slight recovery was observed after a further 100 cycles. This was found to be related to the extent of the structural damage to the coating. The surface remained hydrophobic after 200 cycles, but after an additional 100 cycles the coating had become water permeable, leading to a sharp reduction in WCA and a reduction in roll-off angle. This suggests that damage from abrasion has partially removed the coating, affecting its ability to repel water.

TABLE II - CS10 ABRASION TEST RESUlTS

\begin{tabular}{|c|c|c|c|c|c|}
\hline & Initial & $\begin{array}{c}\mathbf{5 0} \\
\text { cycles }\end{array}$ & $\begin{array}{c}\mathbf{1 0 0} \\
\text { cycles }\end{array}$ & $\begin{array}{c}\mathbf{2 0 0} \\
\text { cycles }\end{array}$ & $\begin{array}{c}\mathbf{3 0 0} \\
\text { cycles }\end{array}$ \\
\hline WCA ( $\left.{ }^{\circ}\right)$ & 118.8 & 107.2 & 105.9 & 101.3 & 66.0 \\
\hline Roll-off angle ( ${ }^{\circ}$ ) & 20.6 & 25.4 & 29.8 & 47.1 & 32.5 \\
\hline R (\%) & 8.2 & 8.0 & 8.1 & 8.2 & 8.1 \\
\hline T (\%) & 91.8 & 92.0 & 91.9 & 91.8 & 91.9 \\
\hline
\end{tabular}

Unlike the CS10, the use of the felt pad as the abrader showed that after the same number of cycles with the same amount of load, the coating WCA did not degrade more than $1 \%$. The optical performance was not affected by either abrasive material. 


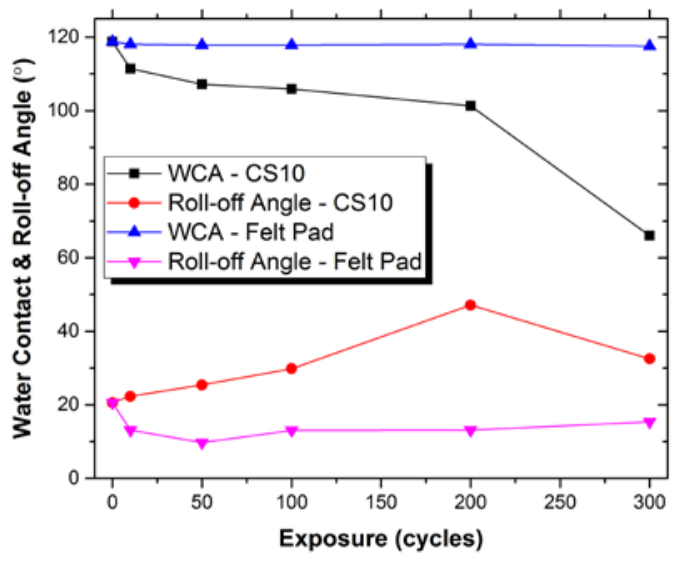

Fig. 6. Hydrophobic performance before and after abrasion with CS10 and Felt Pad.

\section{F. Solubility Test}

The samples were immersed in deionized (DI) water to assess the performance of the coating against exposure to water. The total duration of the test was 1000 hours. The performance of the coating was measured at 50 and 100 hours, and at 200 hour intervals. Figure 7 shows the results during the exposure.

The coating showed exceptional resilience to damage from DI water exposure with only a $3^{\circ}$ drop in WCA and no change in RoA and optical performance. The results confirm that the water repellency of the surface is unlikely to be affected in rainy climates.

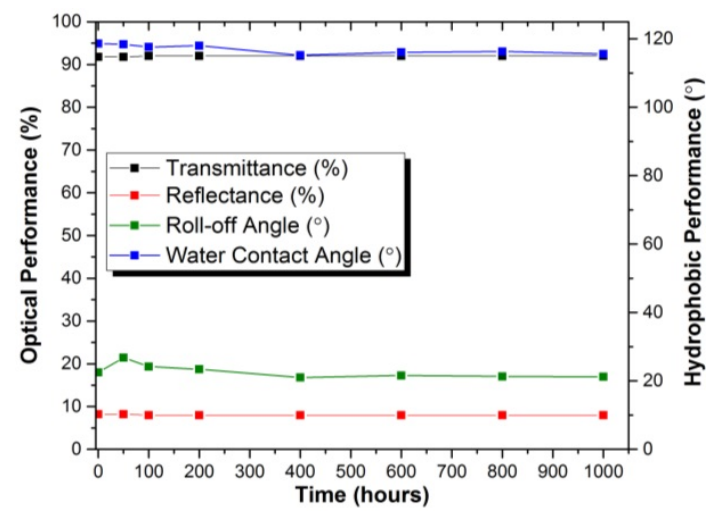

Fig. 7. Optical and hydrophobic performances during DI water immersion.

\section{G. UV Exposure}

The UV Exposure test described in IEC 61215-2:2016 [8] was applied to the coatings. Samples were exposed for 500 hours which is equivalent to $15 \mathrm{kWh} / \mathrm{m}^{2}$ of UV light.
Figure 8 shows the effect of the UV exposure on the coating after 50, 100, 250 and 500 hrs. The WCA lowered to $87.0^{\circ}$ and the RoA increased to $60.5^{\circ}$ after the exposure.

The surface chemical composition was also analysed to observe any corresponding change in the composition. The XPS surface scan was performed on the hydrophobic coating before and after the UV exposure test.

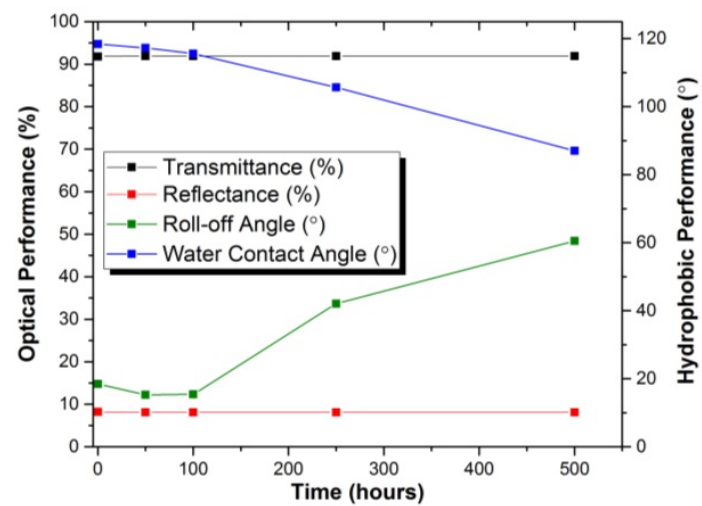

Fig. 8. Optical and hydrophobic performance during UV Exposure.

Figure 9 shows the high resolution XPS scan of the C1s peak. After the UV exposure, a strong reduction in F1s concentration was detected, from $\sim 40$ to $\sim 26$ At\%. The $\mathrm{CF}_{3} / \mathrm{CF}_{2}$ contribution ( $\sim 294-292 \mathrm{eV}$ ) decreased in favour of the C-C/-C-O functional groups ( 286-284.8 eV). This is confirmed by the increase in O1s concentration, from 26 to 34 At\% as seen in Figure 9. This loss of fluorine in the chemistry of the surface corresponds to the reduction in WCA, and the degradation of the hydrophobic properties of the coating.

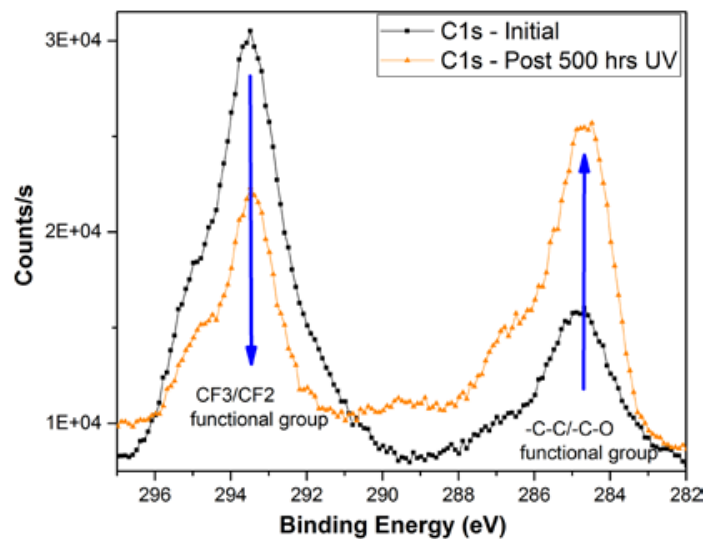

Fig. 9. XPS spectra of the hydrophobic surface before and after 500 hours of UV Exposure.

The hydrophobic performance of the coating was related to the presence of the NPs distributed on the surface of the coating. In fact, the loss of fluorine was found to be directly 
correlated to the reduction of NPs distribution on the surfaces, which eventually reduced to zero, after 500 hours of UV exposure. The SEM image after UV exposure confirms the drastic reduction of NPs on the surface of the coating. The NPs were not visible/detectable on the surface, while the formation of pockets and dark spots occurred, as shown in Figure 10.

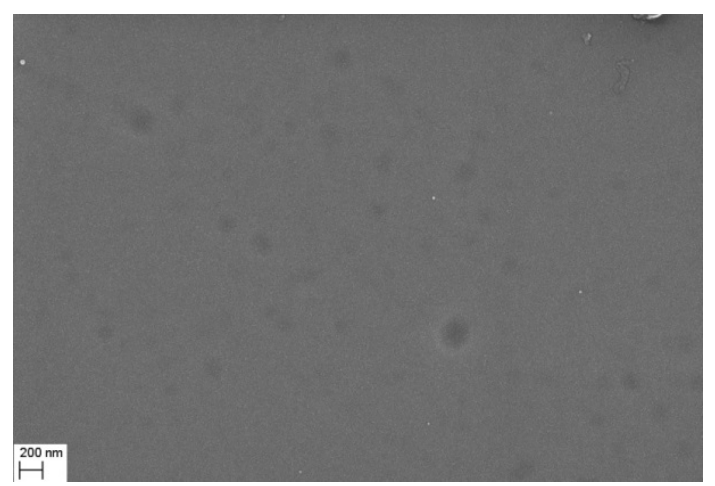

Fig. 10. SEM planar view of the coating after UV exposure.

\section{H. Outdoor Exposure}

Surfaces were exposed to outdoor conditions facing south at an angle of $45^{\circ}$ in Nottingham, UK from July to February. The range of temperatures during this period was $21.3^{\circ} \mathrm{C}$ to $-0.5^{\circ} \mathrm{C}$, a maximum difference of $21.8^{0} \mathrm{C}$. Rainfall varied from 85.1 to $46.2 \mathrm{~mm}$ for each month. Sunshine duration per month ranged from 178.5 to 54.9 hours.

Local weather data showed no extreme conditions that would exceed the indoor stress tests. The optical performance and hydrophobicity of each sample was measured monthly, before and after cleaning. Surfaces were cleaned using deionized water and isopropyl alcohol with a soft tissue without applying force. After six months of exposure, the hydrophobicity was reduced considerably on both cleaned and uncleaned surfaces. The WCA showed $~ 31 \%$ degradation. The RoA was increased by $\sim 64.0^{\circ}$ and measured $84.0^{\circ}$.

Figure 11 shows the change in optical and hydrophobic performance of the cleaned samples, over a period of 6 months. The graph confirms that the hydrophobic performance of the coating was degraded by outdoor exposure. After cleaning, the optical transmittance of the coating remained unchanged.

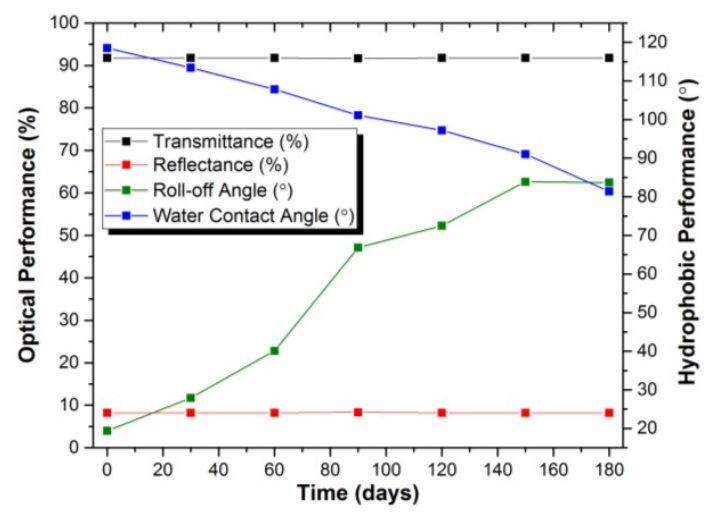

Fig. 11. Optical and hydrophobic performance of outdoor sample.

The hydrophobic surfaces were analysed using SEM and STEM image analysis to observe the changes in NPs distribution after outdoor exposure. Figure 12 confirms the reduction of NPs after outdoor exposure of the hydrophobic surfaces. The NPs seem to be removed by outdoor exposure and the formation of dark spots, similar to those seen in Figure 10, are observed on the surface of the samples in Figure 12a.

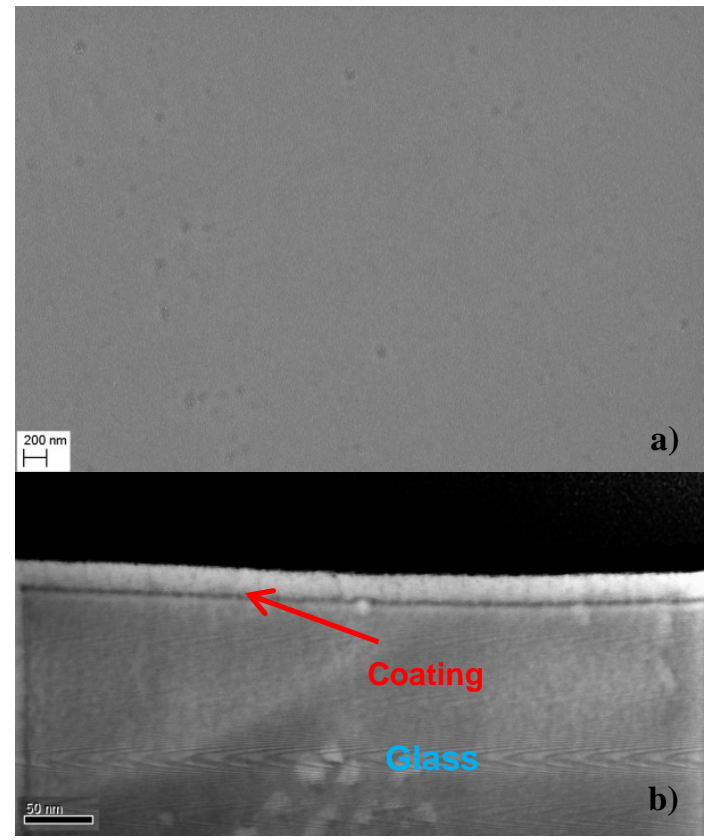

Fig. 12. a) NPs are not visible on hydrophobic coating following outdoor exposure in the SEM planar view image b) STEM image of a coating cross-section with no nanoparticles present.

\section{CONCLUSIONS}

Soiling is one of the most important issues confronting the PV industry. Anti-soiling hydrophobic coatings are becoming commercially available. The anti-soiling effect of the coating depends on the hydrophobic properties. It is important that the hydrophobic performance is maintained for a reasonable time. 
Ideally, this would match the lifetime of the module. However, if this is not technically feasible, the periods between reapplication of the coating must be established. In this paper, we have tested the performance of a hydrophobic coating commonly used in the ophthalmic and display industries and assessed its suitability for use on PV module cover glass.

The coating was exposed to damp heat for a period exceeding the standard PV qualification test. No degradation was observed after 4000 hours of exposure. The coatings showed no degradation in performance after 1000 cycles of temperature cycling test and 10 cycles of humidity freeze test. This durability compares well with well-performing modules.

However, the UV exposure tests reveal that the coating is susceptible to damage on exposure to UV light. Significant degradation in WCA and RoA was observed. This indicates that the coatings may resist the harsh environmental stresses such as damp heat and humidity freeze, but they are susceptible to damage under UV exposure. XPS analysis revealed a clear reduction in fluorine in the composition of the coating after exposure to UV and outdoor testing. SEM analysis revealed that nanoparticles had also been removed. This explains the resulting reduction in contact angle. Outdoor exposure caused degradation in WCA and RoA before and after cleaning. The degradation is likely caused by UV exposure in sunlight correlating with the effects observed in laboratory tests. The hydrophobic coating analysed was not specifically designed for the solar application and resilience to outdoor exposure to sunlight was not part of its specification. It may be that a UV inhibitor could be added to the formulation to enhance its suitability for use on solar modules.

The abrasion test results showed that if the cleaning cycles are performed carefully with soft material e.g. felt pad, the useful performance of the coating can be prolonged. The use of industry standard abrasive materials such as CS10 severely damaged the coating and affected the hydrophobic performance. However, these types of abrasive materials are useful to assess coating durability by comparing the performance of different hydrophobic coatings.

A range of testing methods has been used to assess the performance, environmental stability and wear resistance of a hydrophobic coating previously designed for an alternative application with limited outdoor exposure. The coating was affected by UV exposure and outdoor testing correlated well with the laboratory testing results. The application of hydrophobic coatings on solar cover glass is technically demanding and thorough testing is required with a comprehensive range of testing methods.

\section{REFERENCES}

[1] T. Sarver, A. Al-Qaraghuli, and L. L. Kazmerski, “A comprehensive review of the impact of dust on the use of solar energy: History, investigations, results, literature, and mitigation approaches," Renew. Sustain. Energy Rev., vol. 22, pp. 698-733, 2013.

[2] E. A. Ömer Kesmez, H. Erdem Çamurlu, Esin Burunkaya, "Sol - gel preparation and characterization of anti-reflective and self-cleaning $\mathrm{SiO}_{2}-\mathrm{TiO}_{2}$ double-layer nanometric films,” vol. 93, pp. 1833-1839, 2009.

[3] T. Young, "An Essay on the Cohesion of Fluids,” Philos. Trans. R. Soc. London, vol. 95, pp. 65-87, 1804.

[4] R. N. Wenzel, "Resistance of Solid Surfaces to Wetting by Water,” Ind. Eng. Chem., vol. 28, no. 8, pp. 988-994, 1936.

[5] A. B. D. Cassie and S. Baxter, "Wettability of Porous Surfaces,” Trans. Faraday Soc., vol. 40, no. 5, pp. 546-551, 1944.

[6] K. Isbilir, B. Maniscalco, R. Gottschalg, and J. M. Walls, "Test Methods for Hydrophobic Coatings on Solar Cover Glass,” in 44th IEEE Photovoltaic Specialists Conference, 2017.

[7] K. Tsui, Q. Lin, H. Chou, Q. Zhang, H. Fu, and P. Qi, "Low-Cost, Flexible, and Self-Cleaning 3D Nanocone AntiReflection Films for High-Efficiency Photovoltaics,” Adv. Mater., vol. 26, pp. 2805-2811, 2014.

[8] BSI Standards Publication, “Terrestrial Photovoltaic ( PV ) Modules - Design Qualification and Type Approval Part 2: Test Procedures,” 61215-2-2016, 2017.

[9] ASTM, "Standard Test Method for Abrasion Resistance of Organic Coatings by the Taber Abraser,” D4060-14, 2014. 